\title{
Resolution of Neonatal Hypertrophic Cardiomyopathy Presumed Secondary to Acquired Maternal Ribonucleoprotein and Smith Autoantibodies
}

\author{
A. Shah, BSc, MBBS, MSc ${ }^{1}$ A. Winrow, MSc, MBBS, MRCP, FRCPCH ${ }^{1}$ R. Fulljames, MSc ${ }^{1}$ \\ N. Naqvi, BSc, MBBS, MSc ${ }^{2}$ R. A. Bansal, MBBS 3 A. S. Bansal, FRCPath, FRCP, DM ${ }^{3}$
}

${ }^{1}$ Department of Paediatrics and Immunology, Kingston Hospital, Kingston, Surrey, United Kingdom

2 Department of Paediatric Cardiology, Royal Brompton Hospital,

Address for correspondence A. S. Bansal, FRCPath, FRCP, DM, Department of Immunology, St. Helier Hospital, Wrythe Lane, Surrey, SM5 1AA, United Kingdom (e-mail: Amolak.Bansal@esth.nhs.uk).

London, United Kingdom

${ }^{3}$ Department of Immunology, St. Helier Hospital, Carshalton, Surrey,

Unitied Kingdom

Am J Perinatol Rep 2013;3:91-96.

\begin{abstract}
Keywords

- hypertrophic cardiomyopathy

- neonatal

- lupus

- anti-RNP and anti-Sm antibodies

- maternal transmission

Severe asymmetrical hypertrophic cardiomyopathy without heart block accompanied by neuromuscular hypotonia and feeding difficulties was evident shortly after birth in the second child of a mother with systemic lupus erythematosus who had no indication of gestational diabetes. High-level anti-ribonucleoprotein (RNP) and Smoth (Sm) antibodies arising from transplacental transfer of maternal antibodies were detected in the child's serum. The cardiac abnormalities improved with a commensurate decline in antibody titers. Previously reported cases of neonatal cardiomyopathy with endocardial fibroelastosis have been ascribed to the transplacental transfer of maternal Sjogrens Syndrome (SS) A (Ro) and Sjogrens Syndrome (SS) B (La) antibodies and have been more severe and persistent compared with our patient. We advocate close monitoring of all babies of mothers with systemic autoimmunity for changes in heart rate during pregnancy and signs of heart failure and neuromuscular weakness after delivery.
\end{abstract}

\section{Case Report}

The second child of a Caucasian mother with a history of systemic lupus erythematosus (SLE) was born at term by emergency cesarean section for spontaneous labor with breech presentation. The mother had not required treatment for her SLE during this pregnancy. A previous child, born 4 years' earlier, had been normal in the neonatal period.

During this pregnancy a routine anomaly scan at 22 weeks' gestation was unremarkable. Subsequently an ultrasound scan at 33 weeks' gestation estimated a fetal weight of $3.10 \mathrm{~kg}$ and identified breech presentation. Maternal good health was maintained during pregnancy without symptoms or biochemical evidence of gestational diabetes.

received

December 10, 2012

accepted after revision

March 16, 2013

published online

May 21, 2013
The infant was born in good condition with Apgar scores of 8 at 1 minute and 9 at 5 minutes. Cord blood gas results were normal. The infant developed respiratory distress within 10 minutes of delivery requiring supplemental oxygen to treat hypoxia (oxygen saturations less than $92 \%$ in room air). Cardiovascular examination revealed normal heart sounds and a grade 3/6 pansystolic murmur loudest at the apex radiating throughout the precordium. Four limb blood pressure recordings were normal. A chest X-ray revealed marked cardiomegaly with normal appearances of the lung fields. The infant's growth parameters were above the 98th percentile when plotted on an appropriate growth chart.

Examination confirmed generalized hypotonia without evidence of dysmorphic features.
Copyright ( $\odot 2013$ by Thieme Medical Publishers, Inc., 333 Seventh Avenue, New York, NY 10001, USA. Tel: +1(212) 584-4662.
License terms

10.1055/s-0033-1344003. ISSN 2157-6998.

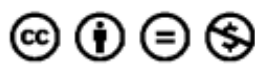


92 Maternal SLE, Resolving Neonatal Hypertrophic Cardiomyopathy Shah et al.

Table 1 Immunologic investigations

\begin{tabular}{|l|l|l|l|l|}
\hline & 3 d postnatal & 25 d postnatal & 3 mo postnatal & 1 y postnatal \\
\hline Rheumatoid factor & $<20$ & & & \\
\hline Ro & 2 & 1 & 2 & 3 \\
\hline La & 4 & 3 & 6 & 32 \\
\hline Sm & 171 & 118 & 85 & 3 \\
\hline RNP & 96 & 85 & $\begin{array}{l}\text { Strongly positive, } \\
\text { speckled }\end{array}$ & $\begin{array}{l}\text { Weakly positive, } \\
\text { speckled }\end{array}$ \\
\hline ANA on HEp2 cells & $\begin{array}{l}\text { Strongly positive, } \\
\text { speckled }\end{array}$ & $\begin{array}{l}\text { Strongly positive, } \\
\text { speckled }\end{array}$ & & 1.24 \\
\hline Complement C3 & & 1.15 & & 0.22 \\
\hline Complement C4 & & 0.25 & & 5.9 \\
\hline IgG & & 4.3 & & 0.25 \\
\hline IgA & & $<0.07$ & & 0.90 \\
\hline IgM & & $<5$ & & \\
\hline IgG anticardiolipin antibodies & & $<12.3$ & & \\
\hline ds DNA antibody & & & & \\
\hline
\end{tabular}

Abbreviations: anti-nuclear antibodies, ANA; ds DNA, double-stranded DNA; IgA, immunoglobulin A; IgG, immunoglobulin G; IgM, immunoglobulin M; Sjogrens Syndrome (SS) A, Ro; Sjogrens Syndrome (SS) B, La; Smith, Sm; human epidermoidcancer, HEp2; ribonucleoprotein, RNP.

The infant's clinical presentation and chest X-ray findings suggested the possibility of underlying congenital heart disease. A hyperoxia test was performed and was normal.

Intravenous antibiotics were administered in line with unit policy for possible peripartum infection in view of the respiratory distress and hypoxia. Cerebrospinal fluid examination identified a mild lymphocytosis without subsequent identification of any microbial pathogens.

ECG showed sinus rhythm without evidence of heart block and left ventricular hypertrophy. Echocardiography demonstrated situs solitus with concordant connections but severe hypertrophic cardiomyopathy with dynamic obstruction of the left ventricular outflow tract and mild mitral regurgitation. The interventricular septal diastolic dimension was $14 \mathrm{~mm}$ and the left ventricular largest dimension on short axis view at papillary muscle level was $8 \mathrm{~mm}$. Based on the echocardiography findings, propranolol was commenced.

Although the baby's oxygen requirement resolved within 48 hours, problems in establishing feeding necessitated placement of a nasogastric tube. The feeding difficulties were considered to be the result of incoordination of suck and swallow mechanisms and the hypotonia.

Repeat echocardiography at 14 days showed a reduction in the interventricular septum diastolic dimension to $7 \mathrm{~mm}$ and resolving mild asymmetrical left ventricular hypertrophy. There was no residual left ventricular outflow tract obstruction and good left ventricular systolic function. The propranolol was discontinued, and discharge home with domiciliary nasogastric tube feeding occurred on day 35 of life.

\section{Immunologic Investigations}

Antibodies to double-stranded DNA (dsDNA), extractable nuclear antigens (ENA) were assayed using the Phadia system and postnatal maternal blood samples confirmed high titers of Smith (Sm) and Ribonucleoprotein (RNP) autoantibodies and a strongly positive speckled anti-nuclear antibodies (ANA) on human epidermoidcancer (Hep2) cells. Antibodies to Sjogrens Syndrome (SS) A (Ro), Sjogrens Syndrome (SS) B (La), dsDNA, organ-specific autoantibodies, immunoglobulin $\mathrm{G}(\mathrm{IgG})$ anti-cardiolipin antibodies, and complement levels of C3, C4 were normal. There were no abnormalities of renal and hepatic function. The infant's immunologic investigation results are summarized in (-Table $\mathbf{1})$.

\section{Subsequent Clinical Progress}

Nasogastric tube feeding was discontinued at 7 weeks. The cardiac murmur had disappeared by 4 months. Echocardiography at 37 weeks showed persistent hypertrophy of the interventricular wall but of reduced severity. Standardized developmental assessment at 3 months and physiotherapy assessment at 4 months were entirely normal and without evidence of residual hypotonia. Further investigations excluded Prader-Willi syndrome and myotonic dystrophy as a cause of her central hypotonia.

Medium-term follow-up at 5 years of age revealed complete resolution of the abnormal interventricular septal hypertrophy with echocardiographic findings now being within normal limits with an interventricular septum of $6 \mathrm{~mm}$. The patient's mother had developed type 2 diabetes 3 years after the birth of our patient.

\section{Echocardiogram Images}

-Figs. 1A and 1B are echocardiogram images from the postnatal period. -Fig. $\mathbf{2}$ is an echocardiogram image of the child at 5 years of age.

\section{Discussion}

Our patient was born to a mother with SLE who was positive for Sm and RNP antibodies. She developed hypertrophic 

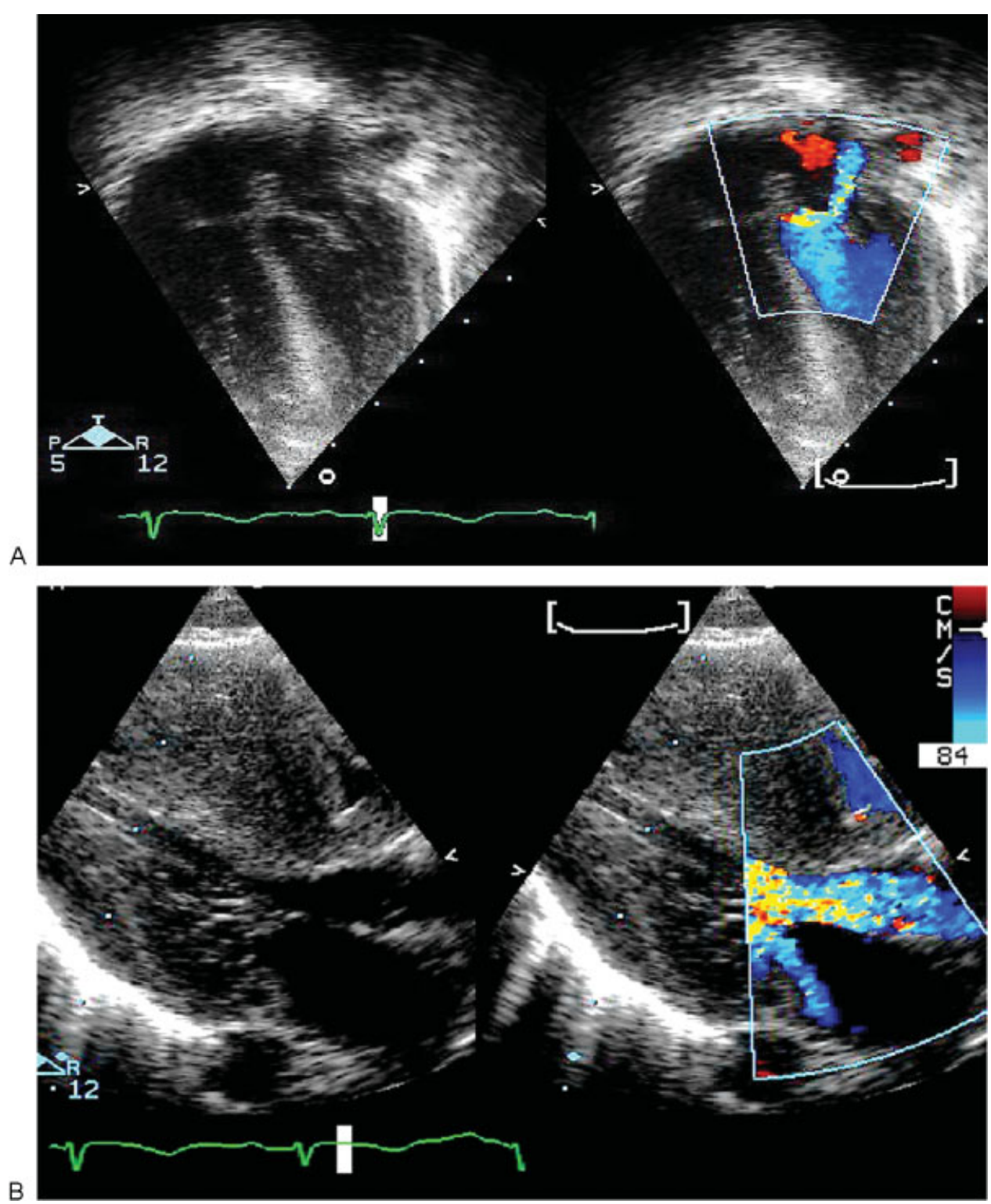

Fig. 1 (A) Echocardiogram of four-chamber view at birth showing left ventricular wall hypertrophy. Color image shows a blue jet of mitral regurgitation (blue arrow). (B) Parasternal long axis showing severe hypertrophy of the interventricular septum (IVS) shown by yellow doubleheaded arrow) causing obliteration of the left ventricular cavity $\left({ }^{*}\right)$. Red arrow shows turbulence of blood flow in left ventricular outflow tract caused by systolic anterior motion (SAM) of the mitral valve.

cardiomyopathy that improved as his level of transplacentally acquired autoantibodies declined. There are two potential causes for the patient's hypertrophic cardiomyopathy: maternal gestational diabetes and autoantibody-mediated disease.

Maternal diabetes is well known to cause neonatal hypertrophic cardiomyopathy. ${ }^{1}$ However, the maternal glucose tolerance test was completely normal and urinalysis throughout the pregnancy was negative. The fact that the child's mother later developed diabetes is certainly interesting. Notwithstanding, it is extremely unusual for such striking septal hypertrophy to occur in the child of a mother with a negative glucose tolerance test.

The hypertrophic cardiomyopathy, hypotonia, and cerebrospinal fluid lymphocytosis evident in our patient are likely the result of transplacental transfer of maternal Sm and RNP antibodies. This is principally suggested by the decline in each of these clinical features with the reduction in level of the maternally transferred autoantibodies. Much of the literature on the effects of transplacentally transferred maternal autoantibodies centers on anti-Ro and anti-La antibodies. In this context postmortem histologic analysis of children dying from anti-Ro-mediated cardiac damage has confirmed fibrosis of the sinoatrial node and bundle of His as the most notable findings. ${ }^{2}$ However, endocardial fibroelastosis (EFE), papillary muscle fibrosis, valvular disease, calcification of the atrial septum, and mononuclear pancarditis were also evident in a significant proportion. ${ }^{2}$ There are few reports on anti-RNP antibodies and fewer still on anti-Sm antibodies in the context of fetal cardiac damage. As such, this report is 
94 Maternal SLE, Resolving Neonatal Hypertrophic Cardiomyopathy Shah et al.

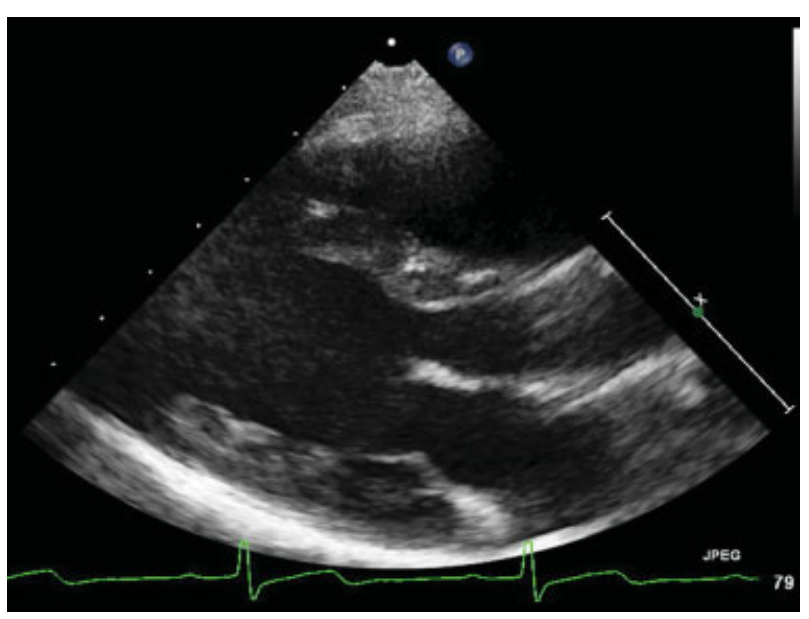

Fig. 2 Echocardiogram of parasternal long axis at 5 years of age. The hypertrophy of the left ventricle has resolved and now there is a normal-sized left ventricular cavity (white double-ended arrow).

relatively unique in showing a good correlation between myocardial interventricular septal wall thickness and autoantibody levels. In contrast to the reduced heart rate that is frequent in anti-Ro- and anti-La-mediated neonatal cardiac disease, there was no disturbance in heart rate or evidence of sinoatrial dysfunction in our patient with maternally transferred anti-RNP and Sm antibodies.

Isolated neonatal complete heart block ( $\mathrm{CHB})$ is infrequent in the absence of anti-Ro and anti-La antibodies. More than 85 to $90 \%$ of mothers having offspring with this problem are reported to have had one or both autoantibodies. ${ }^{3,4}$ In comparison they were present in only $8 \%$ of the mothers with children who had heart block after the neonatal period. ${ }^{11}$ Estimates of the risk of $\mathrm{CHB}$ in fetuses and infants born of mothers with significant anti-Ro and anti-La antibodies has been quoted to be between 1 and $6 \%^{5-7}$ The risk of in utero death from anti-Ro-mediated cardiomyopathy has been estimated to be around $6 \%$ and the cumulative probability of survival at 10 years of age is $86 \%{ }^{8}$

Echocardiography of fetuses of women with lupus have also revealed cases of pulmonary stenosis, tricuspid regurgitation, and, less frequently, mitral regurgitation. ${ }^{9}$ Interestingly, recent work suggests that anti-Ro antibodies may also have a negative impact on the adult heart by prolongation of the QTc interval, possibly resulting from a direct inhibitory interaction with the potassium current $\mathrm{I}(\mathrm{Kr}) .{ }^{10}$ Unfortunately, an increased proportion of children with neonatal lupus syndrome (NLS) and CHB progress to cardiomyopathy and sudden death compared with children with idiopathic nonautoimmune $\mathrm{CHB}{ }^{11}$

It is presently unclear why only a small proportion of children born of mothers with lupus accompanied by Ro and La antibodies develop NLS and cardiac disease in particular. Not infrequently the autoantibodies are not pathogenic in the mother and the maternal lupus is only diagnosed after it is diagnosed in the child. Interestingly neonatal lupus with cutaneous features but not cardiac disease has been de- scribed in one but not the other monozygotic twin even though high-titer anti-Ro antibodies were present in both. ${ }^{12}$ The risk of NLS, and particularly CHB, increases significantly in children born subsequent to an affected child. ${ }^{13}$ This risk is not reported to be affected by maternal health, use of steroids, antibody status, severity of cardiac disease in the first affected child, or sex of the subsequent child. ${ }^{14}$ Recently, Jaeggi et al $^{15}$ have shown the cardiac manifestations of NLS (CHB and EFE) to be more frequent in women with moderate or high anti-Ro antibody levels. CHB is more frequent in women with anti-Ro antibodies associated with hypothyroidism than in those without associated thyroid dysfunction. ${ }^{16}$ Important risk factors for an adverse outcome in $\mathrm{CHB}$ and anti-Ro disease include nonwhite maternal race. ${ }^{8}$ This was due to the higher incidence of EFE and hydrops in the latter.

The mechanism by which anti-Ro and anti-La antibodies mediate neonatal cardiac damage has been recently reviewed by Izmirly et al. ${ }^{17}$ Animal studies suggest that autoantibody reactivity to the p200 region of the Ro52 protein appears to be of significant importance. There is also a considerable role for the autoantibody targeting of L-type calcium channels and in addition in vitro studies suggest an interesting protective role of $\beta-2$ glycoprotein 1 by reducing the binding of the anti-Ro antibodies. ${ }^{17}$ There is little work on anti-Ro antibody avidity and the risk of CHB. How anti-RNP and anti-Sm antibodies produce cardiomyopathy is unclear, although an initial cardiac insult with expression of the appropriate antigens on the surface of myocytes is a possibility as it is with anti-Ro antibodies. ${ }^{3}$ However, the binding of anti-Ro but not anti-DNA, anti-La, anti-Sm, or anti-RNP antibodies from the sera of mothers with infants with $\mathrm{CHB}$ to neonatal but not adult rabbit cardiac tissue and with the ability to alter the transmembrane action potential casts doubt on a direct role. ${ }^{18}$

In regard to autoantibody-mediated cardiac damage without obvious involvement of the conduction system, Nield et al were the first to report infants with EFE arising from maternal anti-Ro and anti-La antibodies. ${ }^{19}$ Two of the three babies succumbed to the cardiac damage, and diffuse myocardial IgG deposition was present in all three. EFE has also been reported in 13 infants with $\mathrm{CHB}$, with more than half of the cases detected prenatally. ${ }^{20}$ Interestingly only one of the mothers had a systemic connective tissue disease (CTD) despite all having anti-Ro antibodies and six having both Ro and La antibodies.

The significantly less severe clinical course and absence of heart block documented in our patient may reflect a decreased capacity of the anti-Sm and RNP autoantibodies to cause detrimental cellular differentiation and cardiac pathology in comparison to anti-Ro and La antibodies. Whether these particular autoantibodies have a lesser ability to activate the complement system is unclear. Atypical features appear to have been evident in other children with neonatal lupus associated with anti-RNP antibodies. ${ }^{21}$ The excellent outcome described in our patient is at variance with the late progression of initially mild heart block in those with anti-Romediated cardiac disease. ${ }^{3}$ 


\section{References}

1 Ullmo S, Vial Y, Di Bernardo S, et al. Pathologic ventricular hypertrophy in the offspring of diabetic mothers: a retrospective study. Eur Heart J 2007;28:1319-1325

2 Llanos C, Friedman DM, Saxena A, et al. Anatomical and pathological findings in hearts from fetuses and infants with cardiac manifestations of neonatal lupus. Rheumatology (Oxford) 2012; 51:1086-1092

3 Buyon JP, Rupel A, Clancy RM. Neonatal lupus syndromes. Lupus 2004; 13:705-712

4 Julkunen H, Miettinen A, Walle TK, Chan EK, Eronen M. Autoimmune response in mothers of children with congenital and postnatally diagnosed isolated heart block: a population based study. J Rheumatol 2004;31:183-189

5 Hornberger LK, Al Rajaa N. Spectrum of cardiac involvement in neonatal lupus. Scand J Immunol 2010;72:189-197

6 Lockshin E, Bonfa K, Elkon and M.L. DruzinNeonatal lupus risk to newborns of mothers with SLE. M.D. Arthritis Rheum. 1988;31: 697-701

7 Ramsey-Goldman R, Hom D, Deng JS, et al. Anti-SS-A antibodies and fetal outcome in maternal systemic lupus erythematosus. Arthritis Rheum 1986;29:1269-1273

8 Izmirly PM, Saxena A, Kim MY, et al. Maternal and fetal factors associated with mortality and morbidity in a multi-racial/ethnic registry of anti-SSA/Ro-associated cardiac neonatal lupus. Circulation 2011;124:1927-1935

9 Krishnan AN, Sable CA, Donofrio MT. Spectrum of fetal echocardiographic findings in fetuses of women with clinical or serologic evidence of systemic lupus erythematosus. J Matern Fetal Neonatal Med 2008;21:776-782

10 Lazzerini PE, Capecchi PL, Acampa M, et al. Arrhythmogenic effects of anti-Ro/SSA antibodies on the adult heart: more than expected? Autoimmun Rev 2009;9:40-44

11 Villain E, Coastedoat-Chalumeau N, Marijon E, Boudjemline Y, Piette JC, Bonnet D. Presentation and prognosis of complete atrioventricular block in childhood, according to maternal antibody status. J Am Coll Cardiol 2006;48:1682-1687
12 Batard ML, Sainte-Marie D, Clity E, Belhabri S, Cotellon P, Pradinaud R. [Cutaneous neonatal lupus erythematosus: discordant expression in identical twins]. Ann Dermatol Venereol 2000;127: 814-817

13 Izmirly PM, Llanos C, Lee LA, Askanase A, Kim MY, Buyon JP. Cutaneous manifestations of neonatal lupus and risk of subsequent congenital heart block. Arthritis Rheum 2010;62:1153-1157

14 Llanos C, Izmirly PM, Katholi M, et al. Recurrence rates of cardiac manifestations associated with neonatal lupus and maternal/fetal risk factors. Arthritis Rheum 2009;60:3091-3097

15 Jaeggi E, Laskin C, Hamilton R, Kingdom J, Silverman E. The importance of the level of maternal anti-Ro/SSA antibodies as a prognostic marker of the development of cardiac neonatal lupus erythematosus a prospective study of 186 antibody-exposed fetuses and infants. J Am Coll Cardiol 2010;55:2778-2784

16 Spence D, Hornberger L, Hamilton R, Silverman ED. Increased risk of complete congenital heart block in infants born to women with hypothyroidism and anti-Ro and/or anti-La antibodies. J Rheumatol 2006;33:167-170

17 Izmirly PM, Buyon JP, Saxena A. Neonatal lupus: advances in understanding pathogenesis and identifying treatments of cardiac disease. Curr Opin Rheumatol 2012;24:466-472

18 Alexander E, Buyon JP, Provost TT, Guarnieri T. Anti-Ro/SS-A antibodies in the pathophysiology of congenital heart block in neonatal lupus syndrome, an experimental model. In vitro electrophysiologic and immunocytochemical studies. Arthritis Rheum 1992;35:176-189

19 Nield LE, Silverman ED, Smallhorn JF, et al. Endocardial fibroelastosis associated with maternal anti-Ro and anti-La antibodies in the absence of atrioventricular block. J Am Coll Cardiol 2002; 40:796-802

20 Nield LE, Silverman ED, Taylor GP, et al. Maternal anti-Ro and antiLa antibody-associated endocardial fibroelastosis. Circulation 2002; 105:843-848

21 Su CT, Huang CB, Chung MY. Neonatal lupus erythematosus in association with anti-RNP antibody: a case report. Am J Perinatol 2001;18:421-426 